Trauma Berufskrankh 2009 · 11[Suppl 1]:71-77 DOI 10.1007/s10039-008-1423-8

(c) Springer Medizin Verlag 2009
A. H. Tiemann · G. O. Hofmann

Funktionsbereich Septische und Rekonstruktive Chirurgie, Klinik für Unfall-

und Wiederherstellungschirurgie, BG-Kliniken Bergmannstrost, Halle, Saale

\title{
Winkelstabile Plattenfixation an der unteren Extremität
}

Das Prinzip der winkelstabilen Osteosynthese an der unteren Extremität ist nicht neu. Dynamische Hüftschraube, dynamische Kondylenschraube, aber auch Winkelplatten sind schon lange fester Bestandteil des Repertoires unfallchirurgischer Techniken [10].

Motiviert durch die Suche nach einer Verringerung des Operationstraumas bei gleichzeitiger Minimierung der durch die herkömmliche Plattenosteosynthese hervorgerufenen Durchblutungsstörungen sowohl an den Knochen als auch an den Weichteilen wurde Mitte der 1980er Jahre das aus der Wirbelsäulenchirurgie bekannte Konzept der winkelstabilen Osteosynthese (WO) mittels eines so genannten Fixateur interne auch an anderen Lokalisationen etabliert [8]. Im Unterschied zu den klassischen Plattenosteosynthesen resultiert die Stabilität der WO nicht aus dem Anpressen der Platte an den Knochen, d. h. durch Haftungsreibungskräfte erzeugende Schraubenzugkräfte [2], sondern definitionsgemäß werden bei der WO die im Knochen verankerten Schrauben so mit einem die Fraktur überbrückenden Kraftträger verbunden, dass sie sich ihm gegenüber nicht in dem Winkel verschieben können, in dem sie befestigt wurden [2]. Aus Sicht der Frakturversorgung handelte es sich dabei um einen regelrechten Quantensprung [11].

Die zunehmende Anwendung dieser Systeme führte zu einem Paradigmenwechsel in der plattenosteosynthetischen Versorgung von Fakturen der langen Röhrenknochen. Galt bis dato die anatomische
Reposition mit weiträumiger Präparation und Darstellung der Fragmente sowie möglichst rigider Stabilisierung als operatives Credo, wich dieses Vorgehen jetzt einer minimalinvasiven „No-Touch-Technik“, bei der die Fraktur durch ein winkelstabiles Implantat unter Beachtung von Achse, Rotation und Länge lediglich überbrückt wird. Das Implantat wird über minimalinvasive Zugänge unter maximaler Weichteilprotektion eingebracht. Die Denudierung der Frakturfragmente entfällt ebenso wie durch die Präparation hervorgerufene Periostalterationen oder Störungen der Mikrozirkulation, da das Implantat nur eine geringe Auflagefläche am Knochen hat („limited contact"). Mikrobewegungen sind bei dieser Osteosynthesetechnik möglich und erwünscht, sie tragen wesentlich zur Stimulation der Frakturheilung bei. Die primäre Frakturheilung wird durch die sekundäre ersetzt.

Ein weiterer Vorteil der so genannten Plattenfixateure ist die Vermeidung von primären (Repositionsverlust oder Fehlstellungen, die aus dem Heranziehen von Fragmenten an die Platte durch eingebrachte Schrauben resultieren) ebenso wie von sekundären Repositionsverlusten (Fragment- bzw. Schraubenauslockerung) [19].

Wurden winkelstabile Implantate an den Extremitäten zunächst vorwiegend bei Femurfrakturen eingesetzt, führten deren Erfolge zu einer immer stärkeren Ausweitung des Indikationsspektrums. Besonderes Interesse galt hierbei einerseits der Versorgung gelenknaher Lä- sionen $[3,10,19,20,32,33]$, andererseits zeigten sich auch bei der Behandlung von Kalkaneusfrakturen positive Ergebnisse $[5,15,35]$.

Analog $\mathrm{zu}$ anderen Lokalisationen sind bei den winkelstabilen Implantaten der unteren Extremität die klassischen geraden von anatomisch vorangepassten Formplatten zu unterscheiden.

\section{Winkelstabile Plattensysteme an der unteren Extremität}

\section{Femur}

Proximales Femur und Femurschaft Insbesondere bei der Versorgung hüftgelenknaher Frakturen ist es durch die Entwicklung und zunehmende Verbreitung der winkelstabilen Plattensysteme zu keinem Paradigmenwechsel gekommen.

Die etablierten Systeme (z. B. PFN und PFN A, $\gamma$-Nagel, DHS, Winkelplatten) bieten neben einer Winkelstabilität ausreichende Möglichkeiten zur sicheren Reposition und Retention der Frakturen und sind in der Regel auch in minimalinvasiver Technik zu implantieren. Dies gilt auch für den subtrochantären Bereich. Er ist, wie im Übrigen auch der Femurschaft, eine Domäne der intramedullären Frakturversorgung (Ausnahmen von dieser Regel werden im Passus „Sonderindikati-

Übernahme aus Trauma und Berufskrankheit $2006 \cdot 8: 228-234$ 


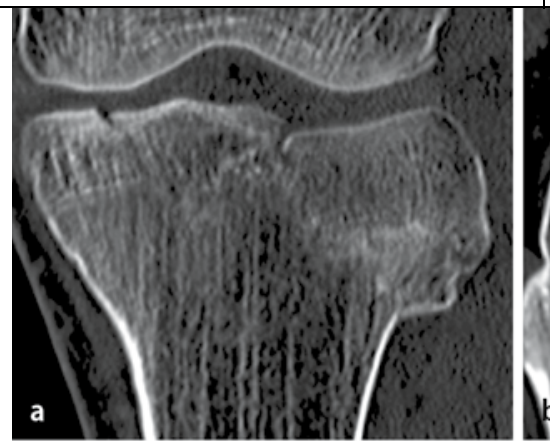

Was gibt es Neues: Innovative Implantate
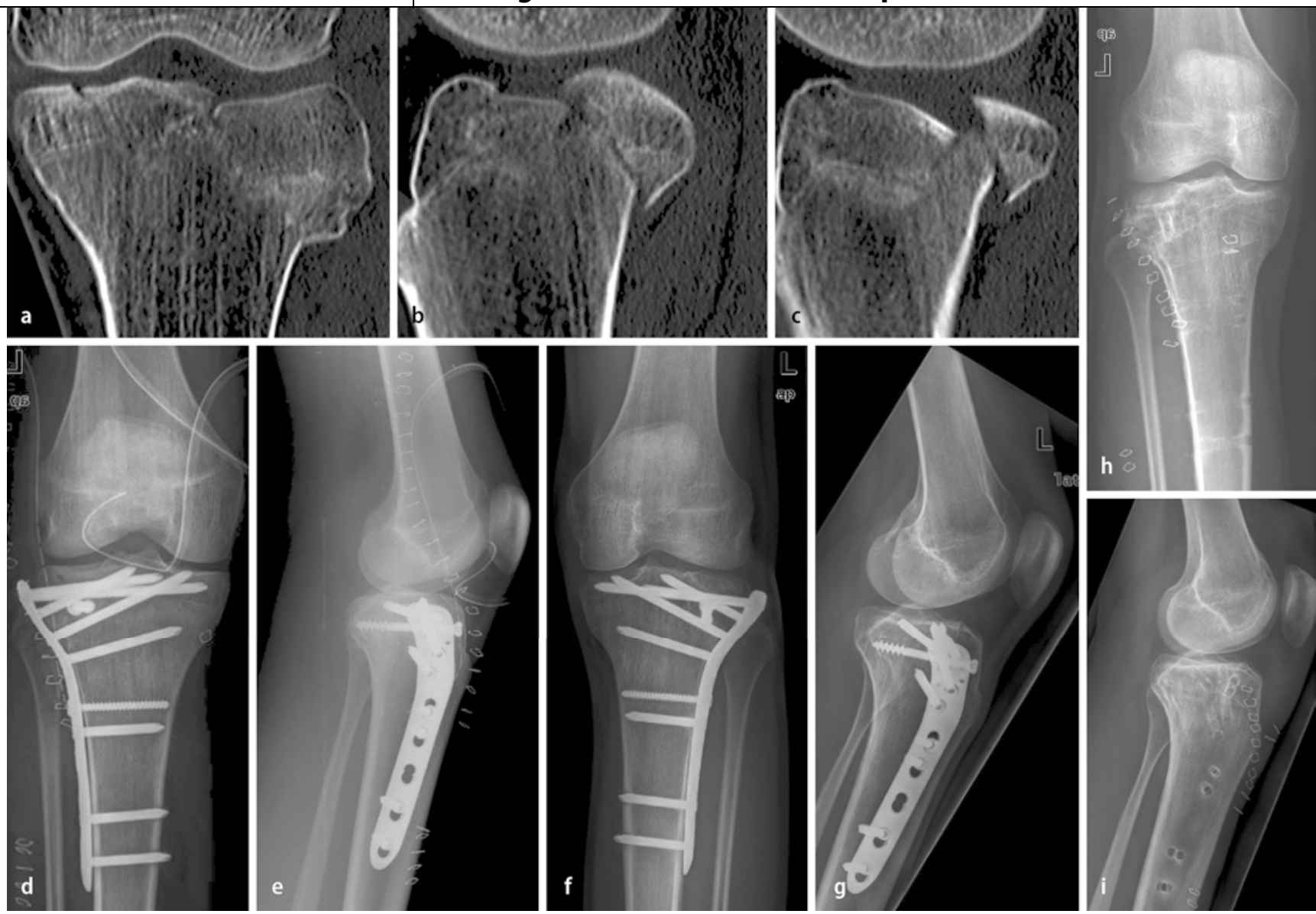

Abb. 1 \ 19-jähriger männlicher Patient, Zustand nach Anpralltrauma am linken Kniegelenk, Tibiakopffraktur,

a-c CT a.-p. und seitlich am Unfalltag, 26.01.2005, d-i Nativröntgenbilder a.-p. und seitlich, d, e postoperativ nach Osteosynthese der Fraktur mit winkelstabiler Formplatte, f,g vom 20.07.2006 mit in anatomisch korrekter Stellung konsolidierter Fraktur, $\mathbf{h}, \mathbf{i}$ vom 23.08.2006 nach Materialentfernung

onen winkelstabiler (Form)platten am Femur" dargestellt) (siehe 0 Praxistipp 1).

\section{Distales Femur}

Die Stabilisierung gelenknaher Femurfrakturen ist aufgrund einer Reihe spezieller Faktoren anspruchsvoll:

- Die Verletzungen sind häufig mit ausgedehnten Weichteilläsionen kombiniert.

- In unmittelbarer Nähe befinden sich wesentliche neurovaskuläre Strukturen.

- Die hier ansetzenden Sehnen und Muskeln erschweren durch ihren Zug die Reposition und Retention der Fraktur.

- Häufig liegen Trümmer- oder Mehrfragmentfrakturen vor $[12,14]$.

Grundsätzlich ist die Versorgung gelenknaher, distaler Femurfrakturen mit intra-, aber auch extramedullären Verfah- ren möglich $[12,17]$. Aufgrund der angeführten Problematik haben sich die körperfernen und gelenknahen Oberschenkelbrüche in den letzten Jahren zunehmend als eine Domäne der winkelstabilen Plattensysteme erwiesen. Das oben bereits erwähnte Prinzip der biologischen Osteosynthese unter maximaler Weichteilprotektion und Schutz von Periost und Mikrozirkulation bietet in diesen z. T. prekären Situationen eindeutige Vorteile gegenüber der klassischen Plattenosteosynthese, aber auch den ,alten“ winkelstabilen Implantaten, wie der DCS oder der 95 -Winkelplatte [3]. In der Regel wird heutzutage LISS („less invasive stabilization system"), ein Formplattensystem, verwendet.

Seine Anwendbarkeit und erste Ergebnisse wurden Ende der 1990er Jahre in einer Multicenterstudie evaluiert [8]. Wong et al. [34] berichteten 2005 insbesondere bei der Versorgung osteoporotischer distaler Femurfrakturen über gute Resultate mit LISS. Schütz et al. [27] führten 2005 eine prospektiven Multicenterstudie über die Versorgung von distalen Femurfrakturen $\left(\mathrm{AO}_{33}\right)$ durch und konnten ebenfalls über positive Ergebnisse berichten. Auch Weight u. Collins [30] kamen zu ähnlichen Resultaten. Sie konnten zeigen, dass mit LISS eine stabile Reposition und Retention bei mechanisch instabilen Rasanztraumen des distalen $\mathrm{Fe}$ murs bei frühzeitiger Vollbelastung zu erreichen war. Vorteilhaft bei der Anwendung von LISS erwies sich auch die Tatsache, dass das System so beschaffen ist, dass aufgrund der wählbaren Plattenlänge auch Femurschaftfrakturen stabilisiert werden können (siehe 0 Praxistipp 2).

\section{Sonderindikationen winkelstabiler (Form)platten am Femur}

Neben den bereits angesprochenen Situationen, in denen sich die Anwendung 
winkelstabiler Implantate anbietet, finden sich zusätzlich spezielle Umstände, bei denen sie von Vorteil sind:

\section{Liegende Hüft- oder Knieendopro-} thesen. Häufig ist eine Versorgung mittels intramedullären Kraftträgern unmöglich.

- Einliegender Hüft- und Knieendoprothese

- Ungünstiger Frakturlokalisation in der Nähe des Prothesenschafts

- Produktion einer Sollbruchstelle, d. h. unmittelbare Nähe von Nagel und Prothesenschaft

In derartigen Situationen kann am proximalen Femur und Femurschaft mit klassischen winkelstabilen Platten und am distalen Femur und Femurschaft mit Formplatten eine belastungsstabile Frakturversorgung erzielt werden (eine ausführliche Diagnostik hinsichtlich des Zustands der liegenden Prothese(n) ist zuvor eine Conditio sine qua non). O'Toole et al. [21] berichteten 2006 über 24 derartige Patienten. Von 19 nachuntersuchten Versorgungen kam es bei 18 zur komplikationslosen Ausheilung. In 1 Fall musste das Implantat durch ein längeres, ebenfalls winkelstabiles ersetzt werden. Wick et al. [31] verglichen die Ergebnisse nach winkelstabiler Verplattung (LISS) bzw. retrograder Nagelung periprothetischer suprakondylärer Femurfrakturen und kamen zu dem Schluss, dass gerade beim osteoporotischen Knochen mit kleinem diaphysärem Fragment LISS Vorteile gegenüber der retrograden Nagelung hat. Auch Kolb

\begin{tabular}{|c|c|}
\hline \multicolumn{2}{|c|}{$\begin{array}{l}\text { Praxistipp } 1 \text { Proximale und Femurschaft- } \\
\text { fraktur }\end{array}$} \\
\hline $\begin{array}{l}\text { Anwendung winkelstabiler Platten- } \\
\text { systeme möglich? }\end{array}$ & Ja! \\
\hline $\begin{array}{l}\text { Anwendung von Formplatten- } \\
\text { systemen sinnvoll? }\end{array}$ & Nein! \\
\hline Implantat der ersten Wahl? & Nein! \\
\hline Implantat für Sonderindikationen? & Ja! \\
\hline
\end{tabular}

\section{Praxistipp 2 Distale gelenknahe Femur-} fraktur

Anwendung winkelstabiler

Plattensysteme möglich?

Anwendung von Formplatten- Ja! systemen sinnvoll?

Implantat der ersten Wahl?

Trauma Berufskrankh 2009 · 11[Suppl 1]:71-77 DOI 10.1007/s10039-006-1190-3

(c) Springer Medizin Verlag 2009

\section{A. H. Tiemann - G. O. Hofmann \\ Winkelstabile Plattenfixation an der unteren Extremität}

\section{Zusammenfassung}

Das Prinzip der winkelstabilen Osteosynthesen ist nicht neu und wurde bereits lange vor der Inauguration winkelstabiler Plattenfixateure erfolgreich beim Fixateur externe, aber auch bei der Stabilisierung von Wirbelfrakturen angewandt. Motiviert durch die Suche nach der biologischen Osteosynthese und unterstützt durch neue Implantatwerkstoffe und Designs war die Umsetzung der den genannten Osteosynthesen zugrunde liegenden Prinzipien bei der Versorgung von Extremitätenfrakturen etwa ab Mitte der 1980er Jahre möglich. Heutzutage weitet sich das

Anwendungsspektrum winkelstabiler Plattensysteme immer weiter aus. Ziel dieses Artikels ist eine Standortbestimmung bei der Versorgung von Frakturen der unteren Extremität. Es werden Möglichkeiten, Sonderindikationen, aber auch die Grenzen des Machbaren in Bezug auf die Anwendung winkelstabiler Plattensysteme an dieser Lokalisation dargestellt.

\section{Schlüsselwörter}

Winkelstabile Plattensysteme .

Untere Extremität · Fuß · Indikationen .

Sonderindikationen

\section{Use of fixed-angle locked-screw plates in fracture treatment in the lower extremity}

\section{Abstract}

The principle of fixed-angle osteosynthesis is not new and had been successfully applied in the external fixators, and also for stabilization of vertebral fractures, long before the new locked-screw plates became available for osteosynthesis of the long bones. During the search for the biological means of osteosynthesis and supported by new designs and materials, the principle of angular stability was extended to plate osteosynthesis in the mid-1980s. The range of indica- tions for locked-screw plates is now becoming wider and wider. The object of this paper is to take stock of the present situation in the treatment of fractures of the lower extremity. Options and special indications are presented, and the limitations of plating systems of this type are also indicated.

\section{Keywords}

Locked-screw plate systems .

Lower extremity · Foot · Indications .

Special indications 
et al. [16] gelang der Nachweis, dass mittels LISS bei liegender Hüft- oder Knieprothese eine suffiziente Frakturversorgung und Vollbelastung nach 6-8 Wochen zu erzielen war. Bei ihrer Untersuchung aus 2006 fanden Kaab et al. [13] bei allen nachuntersuchten Patienten eine knöcherne Konsolidierung ohne sekundären Repositionsverlust und ohne Frühkomplikationen.

Fraktur bei ausgeprägterVarus- oderValgusdeformation und Osteoporose. In derartigen Fällen ist das Einbringen eines Marknagels oft nicht möglich. Zahlreiche Autoren berichteten über die Vorteile der winkelstabilen Implantate, insbesondere beim Vorliegen einer schweren Osteoporose $[26,29,34]$.

Rekonstruktion und Stabilisierung fehlverheilter Frakturen und Pseudarthrosen. Hier stellen die winkelstabilen Implantate eine wesentliche Erweiterung der Palette möglicher Versorgungen dar. Insbesondere die individuelle Anpassung an die Knochensituation, die Möglichkeit auch einer gelenknahen Stabilisierung und der Erhalt der lokalen Durchblutung im kompromittierten Bereich bei sicherer Reposition und Retention sind die wesentlichen Vorteile der winkelstabilen Platten.

\begin{tabular}{l} 
Praxistipp 3 Proximale gelenknahe \\
Tibiafraktur \\
$\begin{array}{l}\text { Anwendung winkelstabiler } \\
\text { Plattensysteme möglich? }\end{array}$ \\
$\begin{array}{l}\text { Anwendung von Formplatten- } \\
\text { systemen sinnvoll? }\end{array}$ \\
\hline Implantat der ersten Wahl? \\
\hline
\end{tabular}

\begin{tabular}{|ll}
\hline $\begin{array}{l}\text { Praxistipp } 4 \text { Tibiaschaftfraktur } \\
\text { Anwendung winkelstabiler Plat- } \\
\text { tensysteme möglich? }\end{array}$ & Ja! \\
$\begin{array}{l}\text { Anwendung von Formplatten- } \\
\text { systemen sinnvoll? }\end{array}$ & Nein! \\
\hline Implantat der ersten Wahl? & Nein! \\
\hline
\end{tabular}

\section{Praxistipp 5 Distale Tibiafraktur/Pilon-} fraktur

Anwendung winkelstabiler Ja! Plattensysteme möglich?

Anwendung von Formplatten- Ja! systemen sinnvoll?

Implantat der ersten Wahl?
Faschingbauer et al. [4] berichteten 2003 über 64 Patienten mit Fehlstellungen und Pseudarthrosen des Femurs: Selbst bei den Problempatienten konnte in allen Fällen eine knöcherne Konsolidierung nach Fixateur-interne-Versorgung erzielt werden (bei atrophen Pseudarthrosen in Kombination mit einer Spongiosaplastik).

\section{Tibia}

\section{Proximale Tibia}

Die anatomischen Gegebenheiten der proximalen Tibia ( $\bullet$ Abb. 1) und die sich daraus ergebenden operativen Konsequenzen sind nahezu deckungsgleich mit denen des distalen Femurs.

Tibiakopffrakturen stellen eine besondere Herausforderung für den Operateur dar. Verschiedene Studien ergaben, dass es nach intramedullärer Stabilisierung proximaler Tibiafrakturen in $44-84 \%$ der Fälle zu Achsabweichungen kommt [6]. Es resultieren im Wesentlichen Valgus-, Antekurvations- und Varusfehlstellungen. Hinzu kommt, dass sich die Maximalvariante der proximalen Tibiafraktur, die Tibiakopffraktur, der Versorgung mit intramedullären Verfahren entzieht.

Neben klassischen Implantaten kommt auch hier LISS zur Anwendung. Die Vorzüge liegen auch hier in der minimalinvasiven Operationstechnik, der mechanischen Stabilität und der sofortigen Belastbarkeit und Beübbarkeit des betroffenen Kniegelenks [22].

Boldin et al. [1] berichteten 2006 über die Versorgung 26 proximaler Tibiafrakturen $\left(\mathrm{AO}_{41} \mathrm{~A}_{2}-\mathrm{C}_{3}\right)$ in einem 3-JahresZeitraum. Hier kam es zu keiner sekundären Dislokation bei extraartikulären Frakturen, bei einer intraartikulären Fraktur kam es zur Varusdeformität. Alle Frakturen heilten knöchern aus. Grützner et al. [7] implantierten LISS bei proximalen Tibiafrakturen 2005 erstmalig computerassistiert. In ihrer Studie von 2004 untersuchten Kääb et al. [12] 29/31 Patienten nach, die zwischen 1998 und 2001 bei proximalen Tibiafrakturen LISS erhalten hatten. Sie beobachteten 1 Plattenlockerung, 2 verzögerte Frakturheilungen und 2 Achsabweichungen über $10^{\circ}$ (siehe - Praxistipp 3).

\section{Tibiaschaft}

Schienbeinschaftbrüche (• Abb. 2) sind auch im Zeitalter der winkelstabilen Plattensysteme Haupteinsatzort für intramedulläre Verfahren (Marknagelung). Durch die Entwicklung neuer Designs mit einer Vielzahl von winkelstabilen Verriegelungsmöglichkeiten konnte die Anwendbarkeit dieser Implantate immer weiter in Richtung der beiden Epiphysen ausgedehnt werden. Heutzutage gelten, je nach Schule, das proximale und das distale Tibiafünftel als Grenze der Marknagelosteosynthese an der Tibia.

Unabhängig davon beschrieben Wolter et al. [33] dennoch Einsatzmöglichkeiten winkelstabiler Plattensysteme am Tibiaschaft. In einer Studie untersuchten sie 55 Patienten, bei denen 57 derartige Eingriffe durchgeführt wurden. Eine Analyse des Patientenguts zeigte, dass es sich hauptsächlich um korrigierende Eingriffe bzw. Verfahrenswechsel nach primärer Stabilisierung im Fixateur externe handelte (26-mal Therapie einer Pseudarthrose, 3-mal Umstellungsosteotomie, 9-mal Umstieg vom Fixateur externe, zusammen $69 \%$ der behandelten $\mathrm{Pa}$ tienten). Nur 21-mal erfolgte die primäre Frakturstabilisierung mittels winkelstabilem Plattensystem. Auch wenn diese Autoren über gute Nachuntersuchungsergebnisse berichteten (kein Korrekturverlust, kein Implantatversagen, komplikationsloser Heilungsverlauf), zeigt die Arbeit dennoch, dass es sich bei der Anwendung dieser Systeme im Schaftbereich der Tibia um eine Sonderindikation handelt (siehe 0 Praxistipp 4).

\section{Distale Tibia}

Analog zu den Ausführungen über distale Femurfrakturen und proximale Schienbeinbrüche kommen auch in dieser Region multiple Faktoren zusammen die Versorgung und Ausheilungsergebnis negativ beeinflussen können ( $\mathbf{A b b}$. 3):

- Geringer Weichteilmantel

- Häufig im Zusammenhang mit Rasanztraumen, d. h. ausgedehnte Weichteilläsionen und Störungen der Mikrozirkulation

- Gelenkfraktur mit Zerstörung des Pilon

- Instabile Fraktursituation 
Gerade im Hinblick auf die Weichteilsituation stellt sich zunächst die Frage, ob eine primäre Versorgung der Verletzung unabhängig von dem dabei verwandten System überhaupt möglich ist oder ob das Prinzip der postprimären oder sekundären Versorgung (primär Fixateur externe, dann Verfahrenswechsel) angewendet werden muss. Wie Mayr [19] 2004 ausführte, konkurrieren dann die Plattenfixateure mit den herkömmlichen Plattensystemen. Die Problematik Letzterer wurde bereits bei den anderen Frakturlokalisationen angesprochen. Gerade in dieser prekären Situation bietet die minimalinvasive Technik der Plattenfixateure deutliche Vorteile. Die Stellung des Gelenkblocks kann zusätzlich durch perkutan implantierte Schrauben erreicht werden, bevor die winkelstabile Platte eingebracht wird. Im Wesentlichen kommen in dieser Lokalisation Formplatten zur Anwendung. Mayr [19] sprach im Bezug auf die distale Tibia-LCP und die Pilon-LCP von einer sinnvollen Bereicherung des traumatologischen Implantatespektrums, deren Anwendung jedoch einer Lernkurve unterliegt [19]. Eine Einschätzung, zu der auch Ryf et al. [24] und Sommer u. Gautier 2003 [28] kamen. Lin et al. [18] fanden bei ihrer Untersuchung von 30 Patienten mit Pilonfrakturen (24 geschlossene, 6 offene Frakturen) nach durchschnittlich $27,1 \mathrm{Mo}-$ naten in $83,3 \%$ gute Ergebnisse mit komplikationslosem Heilungsverlauf. Bei 4 Patienten fanden sich Komplikationen in Form von „Nonunion“, oberflächlichen und tiefen Wundinfektionen. Das eingangs erwähnte zweizeitige Vorgehen bei der Pilonfraktur wurde von Queitsch et al. [23] 2006 untersucht. Nach der Primärversorgung mittels Fixateur externe erfolgte durchschnittlich 8,3 Tage später bei $42 \mathrm{~Pa}$ tienten der Verfahrenswechsel zur winkelstabilen Platten. Radiologisch zeigte sich bei der Nachuntersuchung von 31 Patienten bei 19 keine, bei 9 eine geringe und bei 3 eine fortgeschrittenen posttraumatische Arthrose (siehe $\bullet$ Praxistipp 5).

\section{Sonderindikationen winkelstabiler (Form)platten an der Tibia}

Sie entsprechen denen des Femurs:

- Liegende Knie- oder Sprunggelenkendoprothesen
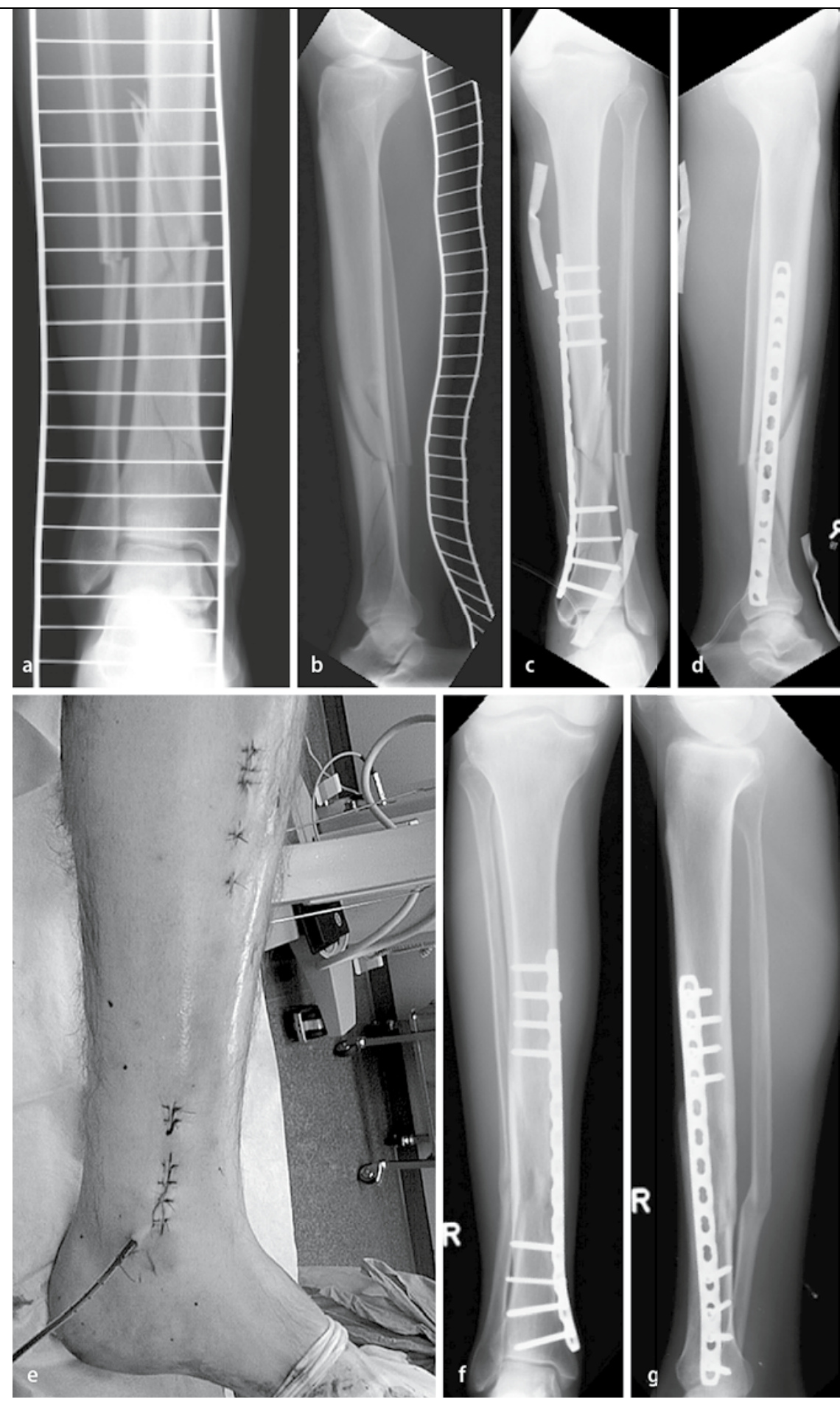

Abb. $2 \triangle$ 31-jähriger männlicher Patient nach Tritt gegen das Schienbein, a-d,f,g Nativröntgenbilder a.-p. und seitlich, a,b vom Unfalltag, $\mathbf{c}, \mathbf{d}$ postoperativ nach Versorgung mit winkelstabiler Platte im Sinne eines Bridging-Systems, Minimalinvasivität des Eingriffs im Sinne der untergeschobenen Platte im intraoperativen Situs ersichtlich (e), $\mathbf{f}, \mathbf{g} 16$ Monate postoperativ knöchern konsolidierte Fraktur

- Fraktur bei ausgeprägter Varus- oder Valgusdeformation und gleichzeitiger Osteoporose der Tibia

- Rekonstruktion und Stabilisierung fehlverheilter Frakturen und Pseudarthrosen
In diesen Fällen gelten die bereits beim Femur analysierten Gegebenheiten und Regeln. 

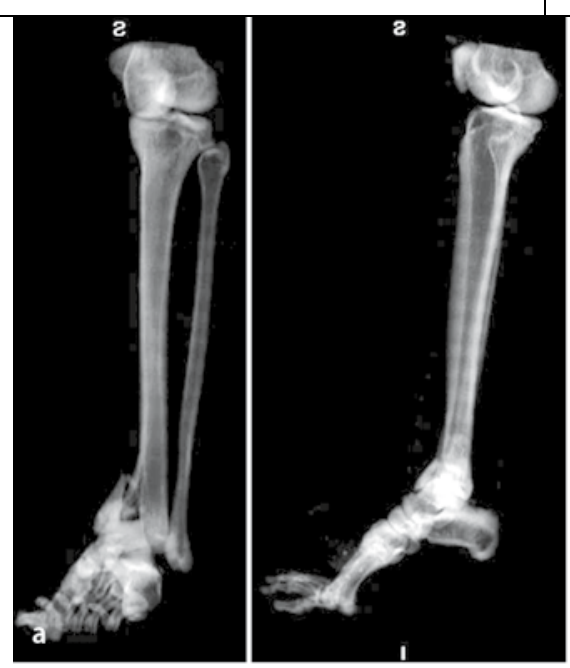

Was gibt es Neues: Innovative Implantate
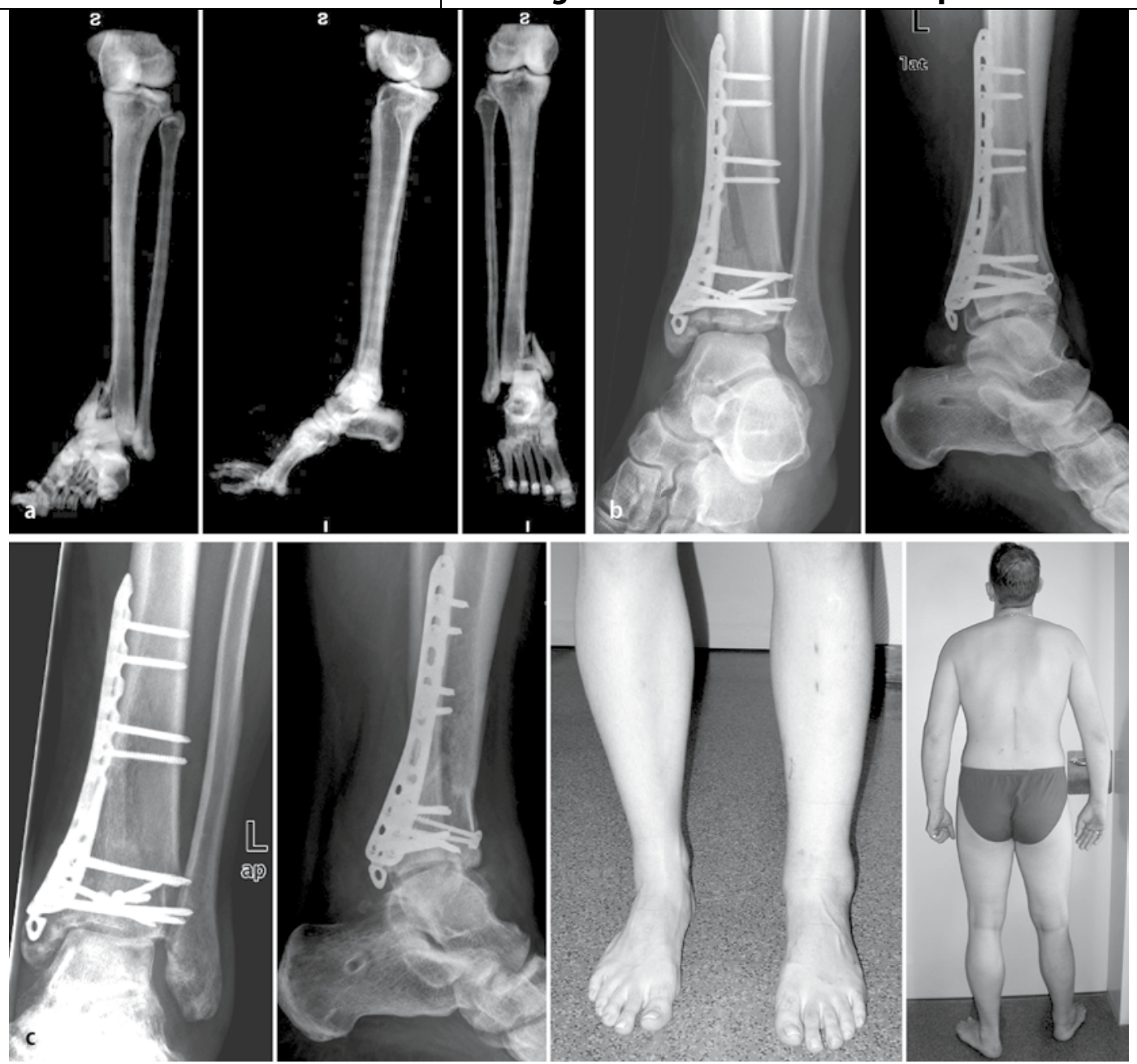

\section{Erfahrungen mit winkelstabilen Plattensystemen am Fuß}

Mit Ausnahme des Kalkaneus bleiben winkelstabile Implantate am Fuß bisher die Ausnahme. Am Kalkaneus kommen sie in den letzten Jahren jedoch zunehmend zum Einsatz.

Will man die spezielle Problematik der Fersenbeinfrakturen betrachten, so ist den Ausführungen von Zwipp et al. [35] aus dem Jahr 2004 nichts hinzuzufügen: „Das Fersenbein ist nicht nur der größte aller tarsalen Knochen, sondern auch der am häufigsten gebrochene. Bei axialer Gewalteinwirkung kann der Calcaneus aufgrund seiner vorwiegend spongiösen Architektur erheblich kollabieren und massiv impaktiert werden. Er ist operativ schwierig aufzurichten, ebenso, wenn nicht gelenkübergreifend, zu retinieren und neigt wegen seiner schmächtigen Weichteilummantelung wie kaum ein anderer Knochen zur Infektion“ [35].
Zahlreiche Autoren haben in der Vergangenheit immer wieder auf die Bedeutung der anatomischen Rekonstruktion des Fersenbeins an sich, aber auch des Kalkaneokuboidal- und des Subtalargelenks verwiesen [25].

Die Literaturangaben über die Wertigkeit winkelstabiler Plattensysteme am Fersenbein sind ambivalent. Nach Zwipp et al. [35] besteht ihr Vorteil potenziell im Verzicht auf eine additive Spongiosaplastik ebenso wie in der Tatsache, dass hochinstabile Situationen (z. B. ausgedehnte B3-Gelenk- und Luxationsfrakturen) sicher retiniert werden können. Die biomechanische Überlegenheit dieser Systeme gegenüber den klassischen Platten hielten die Autoren für noch nicht bewiesen. In ihren Untersuchungen konnten sie jedoch einen signifikanten Rückgang additiver Spongiosaplastiken von $53 \%$ auf $3,8 \%$ beobachten [35]. Kinzl et al. [15] untersuchten $200475 \mathrm{~Pa}$ tienten mit Kalkaneusfrakturen, bei denen ein winkelstabiles Plattensystem implan- tiert worden war. Wie auch im Vergleichskollektiv von 244 mit klassischen Platten versorgten Patienten zeigte sich eine negative Korrelation des postoperativen Outcomes mit der Frakturschwere. Bei 1/3 der Patienten verblieben postoperative Fußabweichungen, $50 \%$ hatten mäßige bis starke Belastungsschmerzen, $12 \%$ zeigten Komplikationen. Die Autoren kamen dennoch zu dem Ergebnis, dass sich eine suffiziente Behandlung der Fersenbeinfrakturen nur mittels winkelstabiler Implantate erreichen lässt [15]. Haustedt et al. [9] untersuchten ebenfalls im Jahr 200449 Patienten nach winkelstabiler Plattenosteosynthese einer Kalkaneusfraktur. Ein sekundärer Korrekturverlust konnte bildmorphologisch bei keinem Patienten nachgewiesen werden, das intraoperative erreichte Ergebnis wurde bis zur knöchernen Konsolidierung der Fraktur gehalten. Komplikationen traten bei 5 Patienten auf [9].

Ungeachtet der Einschätzung von Vorund Nachteilen winkelstabiler Implantate 


\begin{tabular}{|ll}
\hline Praxistipp 6 Kalkaneusfraktur & \\
Anwendung winkelstabiler & Ja! \\
Plattensysteme möglich? & \\
\hline $\begin{array}{l}\text { Anwendung von Spezialplatten- } \\
\text { systemen sinnvoll? }\end{array}$ & Ja! \\
\hline Implantat der ersten Wahl? & Fraglich! \\
\hline
\end{tabular}

am Kalkaneus lässt sich ein Problem bisher nicht beherrschen: Im Gegensatz zu den oben genannten Lokalisationen lassen sich diese Platten nicht minimalinvasiv unter maximaler Weichteilprotektion einbringen. Die klassische Darstellung der Facies lateralis calcanei mit der daraus resultierenden Komorbidität (Nekrosen, Infektionen) ist bisher unvermeidbar. Ebenso bleibt abzuwarten, inwieweit die winkelstabilen Implantate zu einer signifikanten Verkürzung der Entlastungszeit für den betroffenen Fuß führen (siehe Praxistipp 6).

\section{Fazit für die Praxis}

Fasst man die dargestellten Erkenntnisse zusammen, ergibt sich:

1. Die Versorgung von Schaftfrakturen der langen Röhrenknochen der unteren Extremität mittels winkelstabiler Plattensysteme ist zwar prinzipiell möglich, dennoch bleiben diese Verletzungen eine Domäne intramedullärer Verfahren.

2. Je weiter die Verletzung in Richtung Dia-/Epiphyse lokalisiert ist, umso größer werden die Vorteile der winkelstabilen Plattensysteme.

3. Eine Ausnahme von dieser Regel sind die gelenknahen und proximalen Femurfrakturen. Hier bieten die etablierten, ebenfalls winkelstabilen Systeme (DHS, $\gamma$-Nagel usw.) in vollem Umfang die Möglichkeit zur sicheren Reposition und Retention der Frakturen.

4. Bei den angesprochenen Sonderindikationen an Femur und Tibia stellen die winkelstabilen Platten eine wertvolle Erweiterung operativer Optionen dar und sind in der Regel anderen Verfahren sowohl hinsichtlich des operativen Ergebnisses als auch der Komplikationsrate überlegen.

5. Bei Frakturen am Fuß kommen winkelstabile Platten zurzeit nur bei Kalkaneusfrakturen zu Einsatz. Die Aussagen der Literatur zu diesem
Thema sind eher als ambivalent einzustufen. Unstrittig ist, dass der ansonsten so hervorgehobene Vorteil der minimalinvasiven Operationstechnik hier nicht zum Tragen kommt.

\section{Korrespondierender Autor}

\section{PD Dr. A. H. Tiemann}

Funktionsbereich Septische und Rekonstruktive Chirurgie, Klinik für Unfall- und Wiederherstellungschirurgie, BG-Kliniken Bergmannstrost Merseburger Straße 165, 06112 Halle, Saale andreas.tiemann@bergmannstrost.com

Interessenkonflikt. Keine Angaben

\section{Literatur}

1. Boldin C, Frankenhauser F, Hofer HP et al. (2006) Three-year results of proximal tibia fractures treated with the LISS. Clin Orthop 445: 222-229

2. Claes $L$ (2004) Das Prinzip der Winkelstabilität in der Osteosynthese. OP J 20: 4-7

3. Falck M, Höntsch D, Krackhardt T et al. (1999) LISS (less invasive stabilization system) als minimalinvasive Alternative bei distalen Femurfrakturen. Trauma Berufskrankh 1: 402-406

4. Faschingbauer M, Pingen O, Strametz S et al. (2003) Rekonstruktion und Stabilisierung nach fehlverheilten Frakturen und Pseudarthrosen im Femurbereich mit winkelstabilen Implantaten. Trauma Berufskrankh [Suppl 1] 5: 562-566

5. Fuchs S, Wolter D, Kranz HW et al. (2001) Titan-Fixateur-interne-Systeme mit multidirektionaler Winkelstabilität im Unterschenkel- Fuß-Bereich. Trauma Berufskrankh [Suppl 4] 3: 447-453

6. Fuchs S, Wallstabe S, Wenzl ME et al. (2005) Osteosynthese proximaler Tibiafrakturen. Trauma Berufskrankh [Suppl 1] 7: 539-544

7. Grützner PA, Langlotz F, Zheng G et al. (2005) Computer-assisted LISS plate osteosynthesis of proxima tibia fractures: feasibility study and first clinical results. Comput Aided Surg 10: 141-149

8. Haas NP, Schütz M, Hoffmann R et al. (1997) LISS - Less invasive stabilization system - ein neuer Fixateur interne für distale Femurfrakturen. OP J 13: 340-344

9. Haustedt N, Queitsch C, Jürgens C et al. (2004) Neue Möglichkeiten in der operativen Therapie von Fersenbeinfrakturen. Trauma Berufskrankh 6: 176-181

10. Höntzsch D (2004) Erste Erfahrungen mit winkelstabilen Implantaten. Trauma Berufskrankh [Suppl 1] 6: 27-31

11. Hotz T ( 2003) Chirurgie: Winkelstabile Implantate für neue Horizonte in der Frakturversorgung. Schweiz Med Forum 51/51: 12./2003

12. Kääb MJ, Conrad B, Schütz M et al. (2004) Winkelstabile Implantate an der unteren Extremität: das LISSystem zur Versorgung distaler Femurfrakturen und proximaler Tibiafrakturen. OP J 20: 28-31

13. Kaab MJ, Stöckle U, Schütz M et al. (2006) Stabilization of periprosthetic fractures with angular stable internal fixation: a report of 13 cases. Arch Orthop Trauma Surg 126: 105-110

14. Karnezis A, Miles AW, Cunningham JL et al. (1998) "Biological" internal fixation of long bone fractures: a biomechanical study of a "noncontact" plate system. Injury 29: 689-695

15. KinzlL, Arand M, Einsiedel T (2004) Erfahrungen mit winkelstabilen Implantaten am Fersenbein. Trauma Berufskrankh [Suppl 1] 6: 32-34
16. Kolb W, Guhlmann H, Friedel R et al. (2003) Fixation of periprosthetic femur fractures with the less invasive stabilization system (LISS) - a new minimally invasive treatment with locked fixed-angled screws. Zentralbl Chir 128: 53-59

17. Krettek C, Schandelmaier P, Miclau T et al. (1997) Minimally invasive percutaneous plate osteosynthesis (MIPPO) using the DCS in proximal and distal femur fractures. Injury [Suppl 1] 28: A20-A30

18. Lin MS, Lee HM, Hou YT et al. (2003) Treatment of tibial pilon fractures using the Link May Anatomic Bone Plate. J Chin Med Assoc 66: 423-428

19. Mayr E (2004) Winkelstabile Plattenosteosynthese der distalen Tibia. Trauma Berufskrankh [Suppl 2] 6: 258-261

20. Nast-Kolb D, Täger G, Ruchholtz S (2004) Winkelstabile Platten-Schrauben-Verbindungen: distales Femur/proximale Tibia. Trauma Berufskrankh [Suppl 3] 6: 296-301

21. O'Toole RV, Gobzie R, Hwang R et al. (2006) Low complication rate of LISS for femur fracture adjacent to stable hip or knee arthroplasty. Clin Orthop [Epub ahead of print]

22. Petersen W, Zantop T, Raschke M (2006) Tibiakopffraktur. Unfallchirurg 109: 219-234

23. Queitsch C, Kienast B, Fuchs S et al. (2006) Fracture of the distal lower limb: two-stage surgical treatment with external fixator and locked-screw plate. Zentralbl Chir 131: 194-199

24. Ryf C, Gotsch U, Perren T et al. (2003) New surgical treatment procedures in fractures of the distal tibia (LCP, MIPO). Ther Umsch 60: 768-775

25. Sanders R (2000) Displaced intra-articular fractures of the calcaneus. J Bone Joint Surg Am 82: 225-250

26. Schütz M, Südkamp NP (2003) Revolution in plate osteosynthesis: new internal fixator systems. J Orthop Sci 8: 252-288

27. Schütz M, Müller M, Regazzoni P et al. (2005) Use of the less invasive stabilization system (LISS) in patients with distale femoral (AO33) fractures: a prospective multicenter study. Arch Orthop Trauma Surg 125: 102-108

28. Sommer C, Gautier E (2003) Relevance and advantages of new angular stable screw-plate systems for diaphysal fractures (locking compression plate versus intramedullary nail). Ther Umsch 60: 741-756

29. Wagner M, Frenk A, Frigg R (2004) New concepts for bone fracture treatment and locking compression plate. Surg Technol Int 12: 271-277

30. Weight M, Collins C (2004) Early results of the less invasive stabilization system for mechanically unstable fractures of the distal femur (AO/OTA types A2, A3, C2, C3). J Orthop Trauma 18: 503-508

31. Wick M, Müller EJ, Kutscha-Lissberg F et al. (2004) Periprosthetic supracondylar femoral fractures: LISS or retrograde intramedullary nailing? Problems with the use of minimally invasive technique. Unfallchirurg 107: 181-188

32. Wolter D, Schürmann U, Seide K (1999) Universeller Titan-Fixateur interne. Trauma Berufskrankh 1: 307319

33. Wolter D, Fuchs S, Kranz HW et al. (2001) Titanfixateur interne für die Tibia. Trauma Berufskrankh [Suppl 2] 3: 156-161

34. Wong MK, Leung F, Chow SP (2005) Treatment of distal femoral fractures in the elderly using a less-invasive plating technique. Int Orthop 29: 117-120

35. Zwipp H, Rammelt S, Barthel S (2004) Winkelstabile Implantate am Fersenbein, warum? OP J 20: 32-36 